REVISTA CIENCIAS BIOMÉDICAS

ARTÍCULOS ORIGINALES

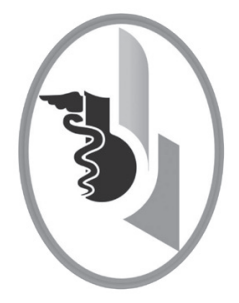

\title{
MANEJO ANALGÉSICO EN PACIENTES CON FRACTURAS DE EXTREMIDADES
}

\author{
ANALGESIC MANAGEMENT IN PATIENTS WITH LIMB \\ FRACTURES
}

\author{
Miranda-Rodríguez Byron $\mathrm{A}^{1}$ \\ Carmona-Lorduys Carlos ${ }^{2}$ \\ Matson-Carballo Gustavo² \\ Montes-Cabarcas Gustavo ${ }^{3}$ \\ Herrera-Banquez Karen $\mathrm{M}^{4}$
}

Correspondencia: byron_mir@hotmail.com

Recibido para evaluación: marzo - 20 - 2015. Aceptado para publicación: octubre - 20 - 2015

\section{RESUMEN}

Introducción: el dolor resultante de una fractura es de difícil control y requiere intervención analgésica así como la inmovilización de la extremidad. No existe en la literatura actual un estudio que compare la intensidad del dolor percibido por el paciente con los diferentes tipos de fracturas, tampoco que especifique qué tipo de manejo inicial se debe realizar para los pacientes con fracturas en extremidades según el tipo de fractura o su clasificación.

Objetivo: determinar la efectividad del manejo analgésico inicial realizado a los pacientes con diagnóstico de fracturas en extremidades, y determinar si existe relación entre el dolor percibido por el paciente, el tipo de fractura y la respuesta analgésica.

Materiales y métodos: estudio observacional, descriptivo y prospectivo para analizar a los pacientes con diagnóstico de fracturas en extremidades atendidos en el Hospital Universitario del Caribe entre los meses de mayo y junio del 2014, se excluyeron pacientes con más de 24 horas de evolución de la fractura, utilización previa a la atención de analgésicos o sustancias psicoactivas, alteración del estado de conciencia, antecedentes de patología neuromuscular o tratamiento analgésico crónico, se utilizó la clasificación AO-ASIF de las fracturas para determinar la localización y tipo de fractura, también se usó la escala verbal del dolor para valorar su intensidad al ingreso y una hora después de postanalgesia. La efectividad de la analgesia fue determinada tomando como efectiva una reducción en $50 \%$ o más del dolor al ingreso.

Resultados: en el período de estudio se identificaron 88 pacientes, de los cuales 21 fueron excluidos por los criterios, en total se analizaron 67 pacientes. Se encontraron diferencias estadísticamente significativas en la percepción del dolor según la evaluación con la escala verbal del dolor en todos los grupos de observación, con medianas de dolor más altas al ingreso y disminución luego de una hora de aplicación del manejo analgésico inicial; sin embargo, solo en el $54.7 \%$ de los pacientes se consideró efectiva la intervención. No se encontró diferencia estadísticamente significativa entre el dolor percibido al ingreso al comparar localización y segmento óseo fracturado, pero si hubo diferencia al comparar la intensidad del dolor según la complejidad del trazo de fractura, siendo mayor el grado de dolor en los trazos de alta complejidad.

1 Médico. Estudiante de postgrado. Ortopedia y Traumatología. Facultad de Medicina. Universidad de Cartagena. Colombia.

2 Médico. Especialista en Ortopedia y Traumatología. Docente. Departamento de Quirúrgico. Facultad de Medicina. Universidad de Cartagena. Colombia.

3 Médico. Especialista en Ortopedia y Traumatología. Hospital Universitario del Caribe. Cartagena. Colombia.

4 Estudiante de Medicina. Facultad de Medicina. Universidad de Cartagena. Colombia. 
ISSN: 2215-7840, 6(2), julio-diciembre 2015, Miranda-Rodríguez Byron A, Carmona-Lorduys Carlos, Matson-Carballo Gustavo, Montes-Cabarcas Gustavo, Herrera-Banquez Karen M

Conclusiones: los analgésicos utilizados para el manejo del dolor en la muestra de pacientes estudiados disminuyeron significativamente el dolor, sin embargo, no lograron una adecuada "efectividad" en el manejo inicial del dolor en los diferentes grupos de fracturas de extremidades. Rev.cienc. biomed.2015;6(2)290-297

\section{PALABRAS CLAVE}

Manejo del dolor; Analgésico; Fracturas óseas.

\section{SUMMARY}

Introduction: pain resulting from a fracture is difficult to control and requires analgesic intervention as well as the immobilization of the limb. There is not available in the current literature a study that compare the intensity of pain perceived by patients with different types of fractures, not specifying what type of initial management should be performed for patients with limb fractures according to the type of fracture. Objective: to determine the effectiveness of initial analgesic management made in patients diagnosed with limb fractures, and to determine if there is a relation among pain perceived by the patient, the type of fracture and the analgesic response. Material and methods: observational, descriptive and prospective study was carried out to analyze patients diagnosed with limb fractures treated at the E.S.E. Hospital Universitario del Caribe between May and June in 2014. Patients were excluded if they had more than 24 hours after onset of fracture, previous use to the attention of analgesics or psychoactive substances, altered state of consciousness, history of neuromuscular disease or chronic analgesic treatment. The AO-ASIF classification of fractures was used to determine the location and type of fracture, and the verbal scale was used to check their pain intensity on admission and an hour after analgesia. The effectiveness of analgesia was determined if the pain was reduced in $50 \%$ or more since admission.

Results: in the study participated 88 patients, 21 patients were excluded by the criteria, a total of 67 patients were identified to be analyzed. Significant differences were identified in perception of pain in all groups, according to the verbal pain scale of observation, median income higher pain was found and there was a decreased 1 hour after application of the initial analgesic management; however, only in $54.7 \%$ of patients the intervention was considered effective. No statistically significant difference was found between perceived pain and fractured bone segment, but it was difference comparing pain intensity according to the complexity of the fracture, with a higher degree of pain in cases of strokes high complexity.

Conclusions: no significant differences were found in pain as the location of the fracture and the bone segments, however there is a difference in the complexity of the fracture line, this is directly related to the energy that causes the fracture, the magnitude of trauma and injury associated with soft tissue. Analgesics used for pain management in the sample of patients studied reduced significantly the pain, however, did not achieve adequate "effectiveness" in the initial management of pain in the different groups of limb fractures. Rev.cienc.biomed. 2015;6(2)290-297

\section{KEYWORDS}

Pain management; Analgesics; Bone fractures.

\section{INTRODUCCIÓN}

El dolor resultante de una fractura es de difícil control y requiere para su manejo intervención analgésica, así como la inmovilización de la extremidad (1). Varios estudios han demostrado la inadecuada administración de los analgésicos, tanto en la elección del medicamento, como en la dosis y frecuencia en que estos se administran a los pacientes con patología dolorosa en los servicios de urgencias $(2,3)$.
El manejo del dolor tradicionalmente no ha sido una prioridad durante la evaluación, el manejo inicial y la reanimación de los pacientes víctimas de trauma músculo-esquelético. De hecho, el uso de analgésicos se ha desalentado en este entorno por desconocimiento, por temor a los efectos adversos o en otros casos por temor a enmascarar, retardar u oscurecer el diagnóstico de lesiones asociadas, aunque la evidencia de esto hasta hace pocos años era pobre o no concluyente (4). 
No existe en la literatura actual un estudio que compare la intensidad del dolor percibido por el paciente, ni el manejo inicial realizado según los diferentes tipos de fracturas. Este estudio pretende determinar, según la localización y complejidad de la fractura, cuál es la intensidad de dolor inicial percibida por los pacientes al ingreso al servicio de trauma en el Hospital Universitario del Caribe, qué tipo de intervención analgésica inicial se realiza actualmente por parte del personal médico encargado del enfoque inicial del paciente fracturado y cuál es la efectividad de dicho manejo.

\section{MATERIALES Y MÉTODOS}

Se realizó estudio observacional, descriptivo y prospectivo en pacientes con diagnóstico de fracturas en extremidades, atendidos en el Hospital Universitario del Caribe entre los meses de mayo y junio del año 2014, fueron excluidos los pacientes que tuvieron tiempo de evolución de la fractura mayor a 24 horas, antecedente de analgesia previa a la atención, consumo de alcohol o sustancias psicoactivas, alteración del estado de conciencia, antecedentes de patología neuromuscular o de tratamiento crónico con analgésicos.

Las variables medidas en cada sujeto fueron el hueso fracturado, segmento óseo fracturado y grado de complejidad del mismo según la clasificación AO-ASIF de las fracturas (5), para la medición de la intensidad del dolor al ingreso se midió una hora analgesia postoperatoria y se utilizó la escala verbal del dolor (VPS) (6), también se midió el tiempo transcurrido entre el ingreso a la urgencia y la administración del analgésico, tipo de analgésico utilizado y vía de administración. La efectividad de la analgesia fue determinada tomando como efectiva una reducción en $50 \%$ o más del documentado en la escala verbal del dolor al ingreso (2-6).

El análisis estadístico de los datos consistió en tablas de frecuencia para las variables cualitativas y estimación de la mediana y recorrido intercuartílico en las cuantitativas por ser de distribución no paramétrica. Como prueba de hipótesis para la comparación de la intensidad del dolor antes y des- pués de una hora analgesia postoperatoria se utilizó el test de Wilcoxon, mientras que para las comparaciones de la intensidad del dolor por grados de complejidad o localización de la fractura se utilizó el test de Mann Whitney, para comparar la proporción de la efectividad de la medicación se utilizó el test exacto de Fisher, un valor de $p<0.05$ fue considerado como significativo.

\section{RESULTADOS}

En el período de estudio se identificaron 88 pacientes ingresados al servicio de urgencias del Hospital Universitario del Caribe con diagnóstico de fractura en extremidades, de estos fueron excluidos ocho por tiempo de evolución de la fractura mayor a 24 horas, cinco por antecedente de analgesia previa a la atención, siete por consumo de sustancias psicoactivas y uno por presentar alteración del estado de conciencia al momento del ingreso, en total se analizaron 67 pacientes.

La mediana de edad de los pacientes fue de 40 años con recorrido intercuartílico entre 26 y 55.5 años, el género masculino fue más frecuente con $62.7 \%$. Los huesos más frecuentemente afectados fueron el cúbito o radio con $49.3 \%$, la tibia o peroné $19.4 \%$, huesos cortos $14.9 \%$, fémur $9.0 \%$ y húmero $7.5 \%$. El segmento óseo más afectado fue el distal con $49.3 \%$, la complejidad de la lesión fue baja en $41.8 \%$, intermedia en $32.8 \%$, y alta en $25.4 \%$ de las fracturas analizadas. El $95.5 \%$ de los pacientes recibió medicación analgésica. El analgésico administrado fue Diclofenaco por vía intramuscular en el $65.7 \%$ de los pacientes, $19.4 \%$ Dipirona y 10.4\% Tramadol, los dos últimos vía intravenosa, el tiempo transcurrido entre el ingreso a la sala de trauma y la administración del analgésico tuvo una mediana de dos horas $(R I=1.5-2)$, (ver Tabla No 1).

Al comparar la intensidad del dolor percibido al ingreso y una hora después luego de recibir el manejo analgésico se encontraron diferencias estadísticamente significativas en la percepción del dolor según la evaluación con la escala verbal del dolor, con medianas de dolor más altas al ingreso, (Gráfica No 1). 
ISSN: 2215-7840, 6(2), julio-diciembre 2015, Miranda-Rodríguez Byron A, Carmona-Lorduys Carlos, Matson-Carballo Gustavo,

Montes-Cabarcas Gustavo, Herrera-Banquez Karen M

\begin{tabular}{|c|c|c|}
\hline \multicolumn{3}{|c|}{$\begin{array}{c}\text { TABLA No } 1 . \\
\text { CARACTERÍSTICAS GENERALES }\end{array}$} \\
\hline & \begin{tabular}{|l|}
$\mathrm{N}$ \\
\end{tabular} & $\%$ \\
\hline Edad Me [RI] & $\begin{array}{l}40[26- \\
55.5]\end{array}$ & - \\
\hline Género masculino & 42 & 62.7 \\
\hline \multicolumn{3}{|c|}{ Hueso afectado } \\
\hline Radio o cúbito & 33 & 49.3 \\
\hline Tibia o peroné & 13 & 19.4 \\
\hline Hueso corto & 10 & 14.9 \\
\hline Fémur & 6 & 9.0 \\
\hline Húmero & 5 & 7.5 \\
\hline \multicolumn{3}{|c|}{ Segmento óseo afectado } \\
\hline Proximal & 9 & 13.4 \\
\hline Medio & 8 & 11.9 \\
\hline Distal & 33 & 49.3 \\
\hline Tobillo & 7 & 10.5 \\
\hline No informado & 10 & 14.9 \\
\hline \multicolumn{3}{|c|}{ Complejidad de la lesión } \\
\hline Baja & 28 & 41.8 \\
\hline Intermedia & 22 & 31.3 \\
\hline Alta & 7 & 10.4 \\
\hline No informado & 10 & 14.9 \\
\hline Medicación & 64 & 95.5 \\
\hline No informada & 3 & 4.4 \\
\hline Diclofenaco $75 \mathrm{mg} \mathrm{IM}$ & 44 & 65.7 \\
\hline Dipirona 2.5 gr IV & 13 & 19.4 \\
\hline Tramadol 50 mg IV & 7 & 10.4 \\
\hline $\begin{array}{l}\text { Tiempo entre el ingreso y } \\
\text { la medicación Me }[\mathrm{RI}]\end{array}$ & $2[1.5-2]$ & - \\
\hline
\end{tabular}

pirona), en los pacientes que no se medicaron la intensidad del dolor no se modificó. En la Gráfica No 2 se muestran los porcentajes de efectividad y no efectividad por analgésico utilizado.

Gráfica $\mathbf{N}^{\circ}$ 2. Efectividad del manejo del dolor por analgésico utilizado

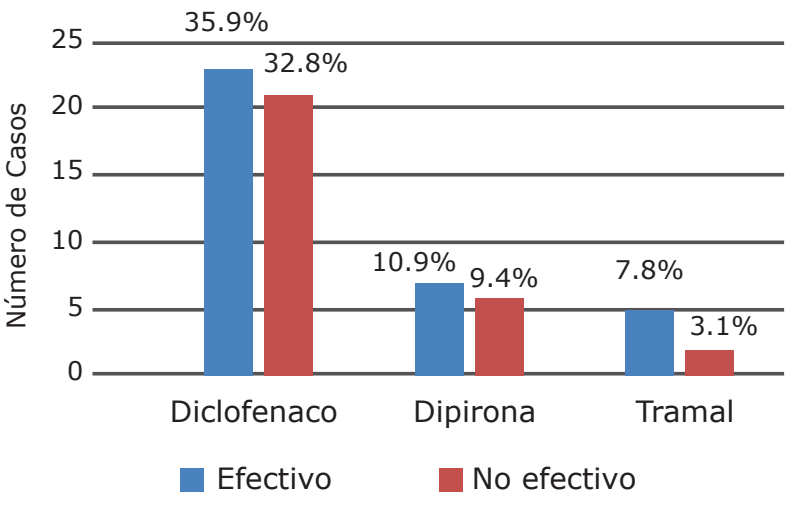

También se comparó la intensidad de dolor según la complejidad en el trazo de fractura, al ingreso y analgesia postoperatoria. Este análisis mostró una disminución significativa de la intensidad del dolor luego de analgesia postoperatoria en todos los grados de complejidad; también se observó mayor intensidad del dolor y mayor disminución de la intensidad del mismo en el grupo de alta complejidad en el trazo de fractura, al ser comparado con el grupo de baja complejidad. Cuando se comparó la proporción de efectividad analgésica ( $\geq 50 \%$ ) entre los grados de complejidad no se observaron diferencias estadísticamente significativas (ver Tabla No 2).

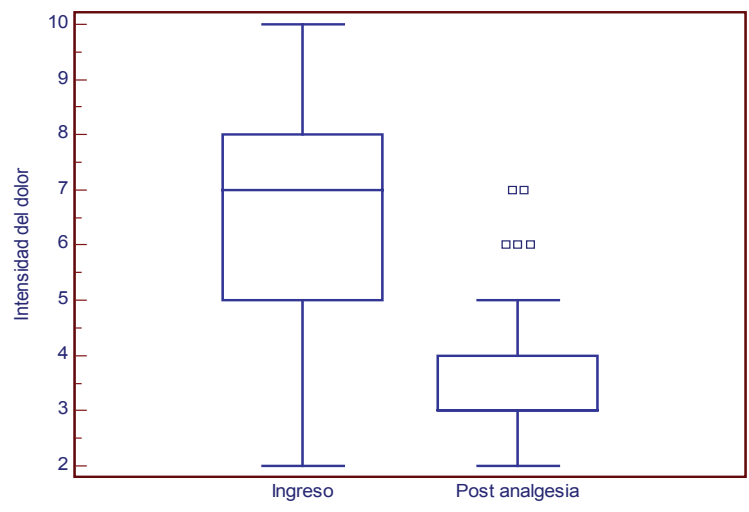

De los 64 pacientes a quienes se le administraron analgésicos en $98.4 \%$ hubo disminución del dolor pero solo en el $54.7 \%$ se consideró efectiva la intervención, solo en un paciente el dolor aumentó (medicado con Di-

Finalmente se realizó el mismo análisis anterior teniendo en cuenta el hueso y el segmento óseo fracturado, observándose de igual manera diferencia significativa en la disminución de la intensidad del dolor luego de analgesia postoperatoria con una única excepción en las fracturas de húmero, pero no se observaron diferencias de la intensidad del dolor al ingreso o posterior a la analgesia postoperatoria entre los diferentes huesos y segmentos óseos afectados (ver Tabla No 3 y No 4).

\section{DISCUSIÓN}

En la actualidad, el acceso a un adecuado tratamiento del dolor es considerado como 
TABLA NO 2.

COMPARACIÓN DE LA INTENSIDAD DEL DOLOR POR COMPLEJIDAD DE LA LESIÓN $\mathrm{n}=\mathbf{5 7}$

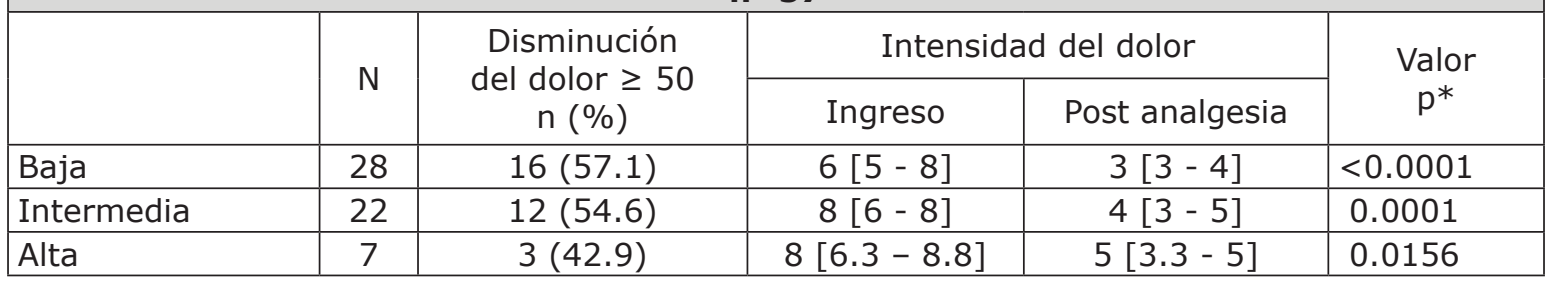

\begin{tabular}{|c|c|c|c|c|c|}
\hline \multicolumn{6}{|c|}{$\begin{array}{c}\text { TABLA No } 3 . \\
\text { COMPARACIÓN DE LA INTENSIDAD DEL DOLOR POR COMPLEJIDAD DEL TRAZO DE } \\
\text { FRACTURA AL INGRESO POSTERIOR AL MANEJO ANALGÉSICO INICIAL } \\
n=67\end{array}$} \\
\hline \multirow{2}{*}{ Hueso afectado } & \multirow[t]{2}{*}{$\mathrm{N}$} & \multirow{2}{*}{$\begin{array}{c}\text { Disminución } \\
\text { del dolor } \geq 50 \\
n(\%)\end{array}$} & \multicolumn{2}{|c|}{ Intensidad del dolor } & \multirow{2}{*}{$\begin{array}{c}\text { Valor } \\
p^{*}\end{array}$} \\
\hline & & & Ingreso & Post analgesia & \\
\hline Radio o cúbito & 33 & $20(60.6)$ & $7[5.8-8]$ & $3[2.8-4]$ & $<0.0001$ \\
\hline Tibia o peroné & 13 & $7(53.9)$ & $6[5-7]$ & $4[3-4.3]$ & 0.0002 \\
\hline Hueso corto & 10 & $4(40.0)$ & $6.5[4-8]$ & $3[2-4]$ & 0.0078 \\
\hline Fémur & 6 & $3(50.0)$ & $6.5[6-8]$ & $3.5[3-4]$ & 0.0312 \\
\hline Húmero & 5 & $1(20.0)$ & $8[5-8]$ & $5[3-6.3]$ & 0.1875 \\
\hline
\end{tabular}

\begin{tabular}{|c|c|c|c|c|c|}
\hline \multicolumn{6}{|c|}{$\begin{array}{c}\text { TABLA No } 4 . \\
\text { COMPARACIÓN DE LA INTENSIDAD DEL DOLOR POR SEGMENTO ÓSEO FRACTURADO } \\
\text { AL INGRESO POSTERIOR AL MANEJO ANALGÉSICO INICIAL } \\
n=57\end{array}$} \\
\hline \multirow{2}{*}{$\begin{array}{c}\text { Segmento del hueso } \\
\text { afectado }\end{array}$} & \multirow[t]{2}{*}{$\mathrm{N}$} & \multirow{2}{*}{$\begin{array}{c}\text { Disminución } \\
\text { del dolor } \geq 50 \\
n(\%)\end{array}$} & \multicolumn{2}{|c|}{ Intensidad del dolor } & \multirow{2}{*}{$\begin{array}{c}\text { Valor } \\
\text { p* }\end{array}$} \\
\hline & & & Ingreso & Post analgesia & \\
\hline Proximal & 9 & $9(55.6)$ & $6[5.8-8.3]$ & $3[2-5]$ & 0.0039 \\
\hline Medio & 8 & $5(62.5)$ & $7[5.5-8]$ & $3[2.5-4.5]$ & 0.0078 \\
\hline Distal & 33 & $18(54.6)$ & $7[5.8-8]$ & $3[3-4]$ & $<0.0001$ \\
\hline Tobillo & 7 & $3(42.9)$ & $8[5.3-8]$ & $4[3.3-4]$ & 0.0156 \\
\hline
\end{tabular}

un derecho humano fundamental y está concebido dentro del tratamiento de las enfermedades e inmerso a su vez, en el derecho a la salud contemplado en la "Declaración Universal de los Derechos Humanos" $(7,8)$.

Los cambios significativos en el abordaje del dolor que se han producido en la última década, y la comprensión de la fisiología del dolor han llevado a las recomendaciones de principios sobre su manejo y a la tendencia de realizar una intervención analgésica agresiva en los pacientes víctimas de trauma con el objetivo no solo de reducir la incomodidad del dolor, sino también para interrumpir el ciclo de dolor provocado por la liberación de hormonas y citoquinas inflamatorias $(9,10)$.
La intervención analgésica adecuada favorece la movilización temprana, períodos más cortos de hospitalización y rehabilitación, disminución de los costos en la atención de los pacientes y lo más importante, aumenta la satisfacción y mejora la evolución de los pacientes con patología traumática (11, 12). Además, disminuye la aparición de los efectos deletéreos del mal manejo del dolor, tales como: el estrés (el cual hace más difícil el manejo del dolor), la alteración de la respuesta inmune que puede llevar a la aparición posterior de infecciones, la alteración de la función cardiopulmonar, la cual ha demostrado incrementar el riesgo de infarto agudo del miocardio y falla respiratoria. Asimismo, disminuye el riesgo de tromboembolismo, 
ISSN: 2215-7840, 6(2), julio-diciembre 2015, Miranda-Rodríguez Byron A, Carmona-Lorduys Carlos, Matson-Carballo Gustavo,

Montes-Cabarcas Gustavo, Herrera-Banquez Karen M

íleo paralitico y todas las morbilidades relacionadas con la inmovilidad y hospitalización prolongada de los pacientes $(13,14,15)$.

En forma general hay una tendencia a la oligoanalgesia en los pacientes tratados por patología traumática, algunos autores reportan que solo aproximadamente el $38 \%$ de los pacientes reciben un adecuado manejo de dolor en el servicio de urgencias $(6,16)$. Estas estadísticas y tendencias hacen que la atención al paciente fracturado y el manejo del dolor secundario a las fracturas represente un reto tanto para las instituciones, como para el personal encargado del manejo de estos pacientes.

En este estudio encontramos que la gran mayoría de los pacientes recibieron algún tipo de manejo analgésico inicial (95.5\%), en todos estos, excepto uno, hubo mejoría al realizar la valoración del dolor una hora posterior a la administración del analgésico; sin embargo, la intervención realizada solo fue efectiva en un $54 \%$ de los pacientes. Este resultado puede ser explicado dado que la elección del medicamento analgésico utilizado en ninguno de los casos se realizó teniendo en cuenta la intensidad del dolor manifestada por el paciente $(3,6)$, ni el mecanismo por el cual se produce el dolor en una fractura, ni sus lesiones asociadas (9, $10,17)$.

Es lógico pensar que como parte de la estrategia para realizar una adecuada evaluación del dolor es necesario implementar por parte del personal encargado de la atención inicial de los pacientes con fracturas, la utilización de una herramienta que se encuentre validada y que permita una valoración lo más objetiva posible de la magnitud del dolor (6). Varios autores han demostrado que la evaluación del dolor de los pacientes es clave para mejorar el manejo del dolor $(18,19)$.

Además, la "Joint Commission on Accreditation of Healthcare Organizations (JCAHO)" reconoció la importancia de la evaluación del dolor y ordenó que la evaluación del dolor se debe llevar a cabo para todos los pacientes que se encuentran bajo cuidado hospitalario (20). La evaluación con la escala verbal del dolor (VPS), es un simple y no invasivo método para evaluar el dolor del paciente en una escala de 0 a 10, ha sido validado y es ampliamente recomendado como la herramienta básica para la evaluación del dolor $(6,20)$.

Se encontró, además, diferencia estadísticamente significativa en la mediana de intensidad del dolor manifestada por los pacientes con trazos de fractura de alta complejidad al ser comparados con los que presentaron fracturas con trazos de baja complejidad. Este resultado tiene que ver con la magnitud del trauma, la cantidad de energía necesaria para causar un trazo de fractura de alta complejidad, y el compromiso de tejidos blandos asociados, comparado con un trazo de baja complejidad.

Donde es entendible que a mayor cantidad de energía, magnitud del trauma y compromiso de tejidos blandos, mayor será el dolor percibido por el paciente, este hecho se puede inferir si se conocen los mecanismos por medio de los cuales se produce el dolor y su fisiopatología (17), pero no existen estudios que relacionen la magnitud de los diferentes tipos de trauma y patrones de lesión, con la medición del dolor que es producida por estos.

Otro hallazgo interesante de este estudio fue que la mayoría de los pacientes 57 (85.1\%) fueron medicados con analgésicos antiinflamatorios no esteroideos (Diclofenaco y Dipirona), medicamentos que se encuentran relacionados con efectos adversos relacionados con la consolidación de las fracturas como retardo en la consolidación y no unión; estos efectos son observados en modelos animales y se han documentado también en pacientes con factores de riesgo para alteraciones de la consolidación (tabaquismo, diabetes, enfermedades metabólicas, enfermedades autoinmunes, desnutrición) (21). Por tal motivo, el uso de este tipo de medicamentos debería hacerse con sumo cuidado y con la evaluación previa del paciente para establecer los factores de riesgo que este pueda presentar en una alteración de la consolidación.

Estos resultados plantean un reto para el personal de atención primaria encargado del manejo analgésico de los pacientes con frac- 
turas, cosa que permite pensar en las posibles estrategias para definir el tipo y dosis de analgésico según la complejidad de la fractura e intensidad del dolor manifestada por el paciente, utilizando herramientas sencillas como el uso de la escala verbal del dolor (VPS) y realizando un adecuado análisis de los mecanismos de trauma, trazos de fractura, y lesiones de tejidos blandos asociados, pero teniendo siempre en cuenta las condiciones y comorbilidades de los pacientes.

Teniendo en cuenta que los analgésicos más utilizados en nuestros pacientes fueron AINES y que fueron los más inefectivos, queda abierta la discusión y debe ser objeto de estudios adicionales y de análisis la utilización de otros analgésicos no utilizados para el manejo del dolor en los pacientes sujetos a este estudio. Dentro de ellos se encuentran los analgésicos pertenecientes al grupo de los opiáceos de alta potencia (morfina, hidromorfona, meperidina, entre otros), los cuales utilizados adecuadamente son seguros, no alteran el proceso de consolidación y se pueden titular en períodos cortos de tiempo, obteniendo una adecuada eficacia analgésica $(11,22)$ y logrando prevenir de esta manera los efectos deletéreos del mal control del dolor que permiten mejorar la satisfacción de los pacientes.

Se recomienda llevar a cabo e impulsar iniciativas académicas encaminadas a optimizar las estrategias que permitan mejorar el manejo analgésico de los pacientes víctimas de trauma con fracturas en extremidades, dichas iniciativas deben basarse en una adecuada evaluación del dolor al ingreso de los pacientes, el conocimiento de la complejidad del mecanismo trauma, trazos de fractura y lesiones de tejidos blandos asociadas. En ese mismo sentido, se debe conocer profundamente los diferentes grupos de analgésicos, su mecanismo de acción, seguridad y las posibilidades que brindan para efectuar una buena titulación para lograr una adecuada eficacia analgésica.

\section{CONCLUSIÓN}

No se encontraron diferencias significativas en el dolor según la localización de las fracturas y los segmentos óseos. No obstante, si hay una diferencia en cuanto a la complejidad de trazo de fractura, esta se relaciona directamente con la energía que causa la fractura, la magnitud del trauma y la lesión de los tejidos blandos asociados. Los analgésicos utilizados para el manejo del dolor en la muestra de pacientes estudiados disminuyeron significativamente el dolor, sin embargo, no lograron una adecuada "efectividad" en el manejo inicial del dolor en los diferentes grupos de fracturas de extremidades.

CONFLICTO DE INTERÉS: ninguno que declarar.

FINANCIACIÓN: recursos propios de los autores.

\section{REFERENCIAS BIBLIOGRÁFICAS}

1. Ekman E, Koman A. Acute pain following musculoskeletal injuries and orthopaedic surgery: mechanisms and management. Bone Joint Surg Am, 2004;86(6):1316-27.

2. Wilson JE, Pendleton JM. Oligoanalgesia in the emergency department. Am J Emerg Med. 1989; 7:620-3.

3. Lewis LM, Lasater LC, Brooks CB. Are emergency physicianstoo stingy with analgesics?. South Med J. 1994; 87(1):7-9.

4. Zohar Z, Eitan A, Halpern $P$, et al. Pain relief in major trauma patients: an Israeli perspective. J Trauma. 2001; 51:767-72.

5. William M. Murphy, Dieter Leu. Clasificación de las fracturas: Significación biológica. Principios de la AO en el tratamiento de las fracturas. 2003;1(4):45-57.

6. Silka Pa. Pain scores improve analgesic administration patterns for trauma patients in the emergency department. Academical Emergency Medicine. 2004; 11(3):264-70.

7. Declaración Universal de los Derechos Humanos, proclamada en el palacio Charcot de París el 10 de diciembre de 1948, ver artículo \# 25.

8. Chóliz M. El dolor como experiencia multidimensional: La cualidad motivacional afectiva. Ansiedad y estrés. No 21. Universidad deValenci abr./1994; 77-88.

9. William J. Phillips, MD, Bradford L. Currier, MD. Analgesic Pharmacology: I. Neurophysiology. J Am Acad Orthop Surg. 2004; 12:213-220. 
ISSN: 2215-7840, 6(2), julio-diciembre 2015, Miranda-Rodríguez Byron A, Carmona-Lorduys Carlos, Matson-Carballo Gustavo, Montes-Cabarcas Gustavo, Herrera-Banquez Karen M

10. William J. Phillips, MD, Bradford L. Currier, MD. Analgesic Pharmacology: II. Specific Analgesics. J Am Acad Orthop Surg. 2004; 12:221-33.

11. Ekman E, Koman A. Acute pain following musculoskeletal injuries and orthopeadic surgery: mechanisms and management. Bone Joint Surg Am. 2004;86(6): 1316-27.

12. Carr DB, Jacox A. Acute pain management: operative or medical procedures and trauma. Clinical practice guideline. Rockville, MD: Agency for Health Care Policy and Research, Public Health Service, US Department of Health and Human Services, 1992 AHCPR publication no. 92-0032.

13. Kehlet $H$. The endocrine-metabolic response to postoperative pain. Acta Anaesthesiol Scand Suppl.1982; 74:173-5.

14. Lewis KS, Whipple JK, Michael KA, Quebbeman EJ. Effect of analgesic treatment on the physiological consequences of acute pain. Am J Hosp Pharm. 1994; 51:1539-54.

15. Chester $\mathrm{C}$, Buckenmaier. The role of pain management in recovery following trauma andorthopaedic surgery. J Am Acad Orthop Surg 2012; 20(suppl 1):s35-s38.

16. Silka PA, Roth MM, Geiderman JM. Patterns of analgesic use in trauma patients in the ED. Am J Emerg Med. 2002; 20:298-302.

17. Norbert P. Sudkamp. Lesiones de los tejidos blandos: fisiopatología e influencia en el tratamiento de las fracturas. Principios de la AO en el tratamiento de las fracturas. 2003;1(5): 59-76.

18. Ricard-Hibon A, Chollet C, Saada S, Loridant B, Marty J. A quality control program for acute pain management in out-of-hospital critical care medicine. Ann Emerg Med. 1999; 34:738-44.

19. Goodacre SW, Roden RK. A protocol to improve analgesia use in the accident and emergency department. J Accid Emerg Med. 1996; 13:177-9.

20. Joint Commission on Accreditation of Healthcare Organizations. JCAHO 2002 Automated Comprehensive Accreditation Manual for Hospitals (CAMH). Available at: http://web.csmc. edu/jcaho/camh/index.html. 2003.

21. Andrew P. Kurmis, BMBS, PhD, Timothy P. Kurmis, BMBS, Justin X. O'Brien, BMBS, and Tore Dal 'en, MD, PhD. The Effect of Nonsteroidal Anti-Inflammatory Drug Administration on Acute Phase Fracture-Healing: A Review. J Bone Joint Surg Am.2012; 94:815-23.

22. Joel E. Holman, MD, Gregory J. Stoddard, MPH, and Thomas F. Higgins, MD. Prescription Opiate in Orthopaedic Trauma. Bone Joint Surg Am. 2013; 95:1075-80.

Universidad de Cartagena Fundada en 1827

REVISTA,CIENCIAS BIOMÉDICAS
Es el órgano de información científica de la Facultad de Medicina de la Universidad de Cartagena. Colombia.

Publique su trabajo en esta revista enviando su manuscrito a: revistacienciasbiomedicas@unicartagena.edu.co www.revistacienciasbiomedicas.com www.revistacienciasbiomedicas.com.co

Revista ciencias Biomédicas es una publicación independiente, imparcial, abierta, revisada por pares, de elevada visibilidad internacional, con circulación online e impresa. Publica artículos en todas las modalidades universalmente aceptadas en inglés y en español, de temas referentes con todas las ciencias biomédicas, incluyendo ámbitos clínicos, epidemiológicos o de estudios básicos.

El sistema de gestión de manuscritos es rápido y justo.

Revista Ciencias Biomédicas está incluida en varias bases de datos latinoamericanas e internacionales.

Antes de enviar su manuscrito, revise las recomendaciones para los autores, presentes en: www.revistacienciasbiomedicas.com www. revistacienciasbiomedicas.com.co 